S. Takenaka

Nagoya Math. J.

Vol. 105 (1987), 19-31

\title{
REPRESENTATION OF EUCLIDEAN RANDOM FIELD
}

\author{
SHIGEO TAKENAKA
}

P. Lévy introduced a notion of Brownian motion $\mathscr{X} \equiv\{X(p) ; p \in M\}$ with parameter in a metric space $(M, d)$, which is a centered Gaussian system satisfying

$$
E|X(p)-X(q)|^{2}=d(p, q) \text { and } X(O)=0, O \text { being the origin . }
$$

In the case of $M=\boldsymbol{R}^{n}, S^{n}$ or the hyperbolic space $\boldsymbol{H}^{n}$ with usual geodesic metric, the Brownian motion above has the following representation

(1) $X(p)=Y\left(S_{p}\right)$, where $S_{p}=\{$ hyperplanes intersect $\overline{O p}\}$ and $\mathscr{Y}=$ $\{Y(\cdot)\}$ is the Gaussian random measure associated with a certain measure $\mu$ on the set of all hyperplanes.

In this paper we shall discuss Brownian motion that corresponds to a general metric. When the metric $d$ on $\boldsymbol{R}^{n}$ is expressible as $d(p, q)=$ $r(|p-q|)$ where $r$ is a positive increasing continuous function, the Brownian motion is called a Euclidean random field (ERF). The main purpose of this paper is to establish the representations of the form (1) for some important classes of ERFs.

In Section 1 we will consider a simple and basic class of ERFs, denote it by $\left\{\mathscr{U}^{\rho}\right\}$, and their representations. The covariance function of the field $\mathcal{U}^{\rho}$ is of finite range and rotationally invariant. The form (1) for the ordinary Brownian motion with parameter $\boldsymbol{R}^{n}$ is obtained as the limit $\rho \rightarrow \infty$ of these fields $\mathscr{U}^{\circ}$.

In Section 2 the representation of type (1) will be considered for general ERF related to the $\left\{\mathscr{U}^{\circ}\right\}$. We will start with a special class of ERFs. If $r(t)=t^{\alpha}$ the random field is called the self-similar Euclidean random field (SERF) of index $\alpha$. The representation of SERF of index $0<\alpha<1$ will be written as a superposition of the fields $\mathscr{U}^{\circ}$. For general ERF, two sufficient conditions for the existence of the representation of form (1) will be given as conditions on the function $r(\cdot)$.

Received June 19, 1985. 
In Section 3, invariance properties of SERFs will be considered in connection with our representations.

\section{§ 0. Introduction}

0-1. P. Lévy defined a notion of Brownian motion with parameter space $M$ equipped with a metric $d$ as below:

Definition 1. A Gaussian system $\mathscr{X}=\{X(p) ; p \in M\}$ is called a Brownian motion with parameter space $(M, d)$ if it satisfies

$$
\begin{aligned}
& X(O)=0 \text { for a fixed origin } O \in M, \\
& X(p)-X(q) \stackrel{\mathscr{s}}{=} N(0, d(p, q)),
\end{aligned}
$$

that is the left-hand-side is subject to the Gaussian law with mean 0 and variance $d(p, q)$.

It is well known that the Brownian motion exists if and only if the covariance function $\sigma(p, q)=(1 / 2)(d(p, O)+d(q, O)-d(p, q))$ is positive definite. In this line, several investigations have been done on the positive definiteness of function $\sigma$ ([3], [6], [7], [9], [10]). In the case of $M=\boldsymbol{R}^{n}$ with usual metric, N. N. Chentsov took more direct manner to obtain Brownian motion in [1] (P. Lévy also treated the case $M=S^{n}$, by the same manner in [5]). To describe Chentsov's method, we need the notion of Gaussian random measure attached to measure space $(E, \mathscr{B}, \mu)$.

Definition 2. A Gaussian system $\mathscr{Y}=\{Y(B) ; B \in \mathscr{B}, \mu(B)<\infty\}$ is called a Gaussian random measure associated with $(E, \mathscr{B}, \mu)$ if it satisfies

$$
\begin{aligned}
Y(B) \stackrel{\mathscr{\varphi}}{=} N(0, \mu(B)) & \\
Y\left(B_{1} \cup B_{2}\right) & =Y\left(B_{1}\right)+Y\left(B_{2}\right), \text { a.e., if } B_{1} \cap B_{2}=\phi .
\end{aligned}
$$

Let $\mathscr{H}$ be the set of all hyperplanes of codimension 1 in $\boldsymbol{R}^{n}$. $\mathscr{H}$ is parametrized by $(q, r) \in S^{n-1} \times \boldsymbol{R}_{+}$as $\mathscr{H} \ni H=\{y ;\langle y, q\rangle=r\}$, and define a measure $\mu$ on $\mathscr{H}$ as $d \mu=d q \cdot d r$, where $d q$ is the normalized uniform measure on $S^{n-1}$. Set

(12) $S_{p}=\{H \in \mathscr{H} ; H$ intersects the line segment $\overline{O p}\}$, then Chentsov's representation of the Brownian motion is

(1) $X(p)=Y\left(S_{p}\right)$, where $\mathscr{Y}$ in the definition 2 is the random measure associated with $(\mathscr{H}, \mu)$. 
0-2. In the case of $M=S^{n}$ and $H^{n}$ - the $n$-dimensional hyperbolic space - the similar results were obtained ([10]):

Let $\mathscr{H}$ be the set of all hyperplanes of codimension 1 in $M$. There exists a canonical measure $\mu$ on $\mathscr{H}$ in the sense of integral geometry. Define a map $S$ from $M$ into the Borel sets of $\mathscr{H}$ as

$$
S_{p}=\{\text { hyperplanes intersect } \overline{O p}\} \text {. }
$$

Then,

$$
X(p)=Y\left(S_{p}\right)
$$

is the Brownian motion with parameter $(M, d)$, where $\mathscr{Y}=\{Y(\cdot)\}$ is the Gaussian random measure associated with $(\mathscr{H}, \mu)$.

The essential parts of the representations above are

i) the pair $\{(E, \mathscr{B}, \mu), S\}$ of measure space and the map $S$. from the metric space $M$ to $\mathscr{B}$.

ii) the relation $d(p, q)\left(=E|X(p)-X(q)|^{2}\right)=\mu\left(S_{p} \triangle S_{q}\right)$ between the metric and the measure, where $\triangle$ means the symmetric difference.

In this paper we will discuss the case of $M=R^{n}$ with rotational invariant $d$ and will establish the pairs $\{(E, \mathscr{B}, \mu), S\}$ for some important classes of random fields.

\section{§1. Random field $\mathscr{U}^{\rho}$ and the Chentsov representation}

We start with a simple class $\left\{\mathscr{U}^{\circ}\right\}$ of Brownian motions and their representations. The original Chentsov's representation will be obtained as the limit $\rho \rightarrow \infty$ of $\mathscr{U}^{\rho}$. Moreover, in the next section the field $\mathscr{U}^{\rho}$ will be used as the basic element to construct representations of certain classes of Brownian motions. In this sense $\left\{\mathscr{U}^{\rho}\right\}$ is an important and fundamental class of Brownian motions.

1-1. Let $M$ be a set and $\mathscr{Y}$ be the Gaussian random measure associated with a measure space $(E, \mathscr{B}, \mu)$. Assume that there is a map

$$
S: M \ni p \longrightarrow S_{p} \in \mathscr{B} \text {. }
$$

Define a Gaussian system

$$
\mathscr{X}=\left\{X(p) \equiv Y\left(S_{p}\right) ; p \in M\right\} .
$$

Then, since $(A \triangle B) \cup(B \triangle C) \supset(A \triangle C)$, the function $d(p, q) \equiv$ $E|X(p)-X(q)|^{2}=\mu\left(S_{p} \triangle S_{q}\right)$ is a pseudo-metric on $M$.

We call this representation a Chentsov type representation of the random field $\mathscr{X}$ with parameter $(M, d)$. 
1-2. The class $\left\{\mathscr{U}^{\rho}\right\}$. Let $\mathscr{Y}=\{Y(B) ; B \in \mathscr{B}\}$ be the Gaussian random measure with respect to $\left(\boldsymbol{R}^{n}, \mathscr{B}, d x\right)$. Set,

$$
S_{p}^{\rho} \equiv\left\{x \in \boldsymbol{R}^{n} ;|x-p| \leqq \rho\right\},
$$

for a positive constant $\rho$, and define a Gaussian system

$$
\tilde{\mathscr{U}}^{\rho} \equiv\left\{\tilde{U}^{\rho}(p) \equiv Y\left(S_{p}^{\rho}\right)\right\}
$$

Then,

Proposition 1. $\tilde{\mathscr{U}}^{\circ}$ is a Gaussian random field stationary under the action of the group $M(n)$ of $n$-dimensional Euclidean solid motions.

Let us calculate the metric function $d^{\rho}$ related to $\tilde{\mathscr{U}}^{\rho}$. Set

$$
r_{\rho}(|p-q|) \equiv d^{\rho}(p, q) \equiv E\left[\tilde{U}^{\rho}(p)-\tilde{U}^{\rho}(q)\right]^{2}=\left|S_{p}^{\rho} \triangle S_{q}^{\rho}\right| .
$$

Then,

$$
r_{\rho}(t) \equiv 4 \sigma_{n-1} \int_{0}^{\min (t / 2, \rho)}\left(\rho^{2}-x^{2}\right)^{(n-1) / 2} d x,
$$

where $\sigma_{n-1}$ is the volume of the $(n-1)$-dimensional unit ball.

Proposition 2.

1) $r_{\rho}(t)=\rho^{n} r_{1}\left(\frac{t}{\rho}\right)$,

2) $r_{1}(t)=O(t)$ when $t \rightarrow 0$,

3) $r_{1}(t)=2 \sigma_{n}$, if $t \geqq 1$,

4) $r_{\rho}(t)=O\left(\rho^{n-1}\right)$ when $\rho \rightarrow \infty$.

It is easy to see that the centered random field

$$
\mathscr{U}^{\rho} \equiv\left\{U^{\rho}(p) \equiv \tilde{U}^{\rho}(p)-\tilde{U}^{\rho}(O)\right\}
$$

is the Brownian motion corresponding to the metric $d^{\rho}(p, q) \equiv r_{\rho}(|p-q|)$.

1-3. Chentsov representation as the limit of $\mathscr{U}^{\rho}$. We note that

$$
\begin{aligned}
r_{\rho}(t) / \rho^{n-1} & =4 \rho \sigma_{n-1} \int_{0}^{\min (t / 2 \rho, 1)}\left(1-x^{2}\right)^{(n-1) / 2} d x \\
& =2 \sigma_{n-1} \cdot t+o(1), \quad \text { when } \rho \longrightarrow \infty .
\end{aligned}
$$

It is natural to expect that the original Chentsov's representation of usual Brownian motion would be obtained as a limit of representations of $\mathscr{U}^{p}$. In fact, this is the case as is shown below. Take a normalization of the measure in $1-2$ as to be 


$$
d \tilde{\mu}_{\rho} \equiv \frac{d x}{2 \sigma_{n-1} \rho^{n-1}} \quad \text { or } \quad=\frac{r^{n-1}}{2 \cdot \rho^{n-1}} d r \cdot d q
$$

in the polar coordinate $x=r \cdot q, r \in \boldsymbol{R}_{+}$and $q \in S^{n-1}$. Related to this normalization we have a series of Gaussian system

$$
\overline{\mathscr{U}}^{\rho}=\left\{U^{\rho}(p) / \sqrt{2 \sigma_{n-1} \rho^{n-1}}\right\}, \quad \rho>0 .
$$

Set,

$$
\overline{\mathscr{U}}_{R}^{\rho}=\left\{\bar{U}^{\rho}(p) ;|p| \leqq R\right\} \quad \text { for fixed } R>0 .
$$

For $\rho>R$, express the set $S_{p}^{\rho} \triangle S_{o}^{\rho}$ in the polar coordinate;

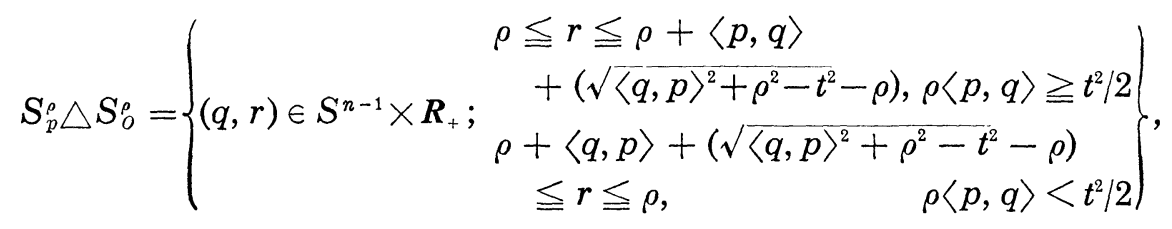

where $t=|p|$.

The term $\left(\sqrt{\langle q, p\rangle^{2}+\rho^{2}-t^{2}}-\rho\right)$ tends to zero when $\rho \rightarrow \infty$. So, let us substract the offset $\rho$ from the radial part $r$ and set $\tilde{r} \equiv r-\rho$. Then,

$$
S_{p}^{\rho} \triangle S_{o}^{\rho}=\left\{(q, \tilde{r}) \in S^{n-1} \times[-R, R] ; \begin{array}{l}
0 \leqq \tilde{r} \leqq\langle q, p\rangle+o(1), \rho\langle p, q\rangle \geqq t^{2} / 2 \\
o(1)+\langle p, q\rangle \leqq \tilde{r} \leqq 0, \rho\langle p, q\rangle<t^{2} / 2
\end{array}\right\} .
$$

On the other hand, for a Borel subset $A$ of $S^{n-1} \times[-R, R]$,

$$
\tilde{\mu}_{\rho}(A)=\mu_{\infty}(A)+o(1), \quad \text { where } d \mu_{\infty}=\frac{1}{2} d q d \tilde{r} .
$$

Finally, set

$$
\chi_{S_{p}}(q, \tilde{r}) \equiv \lim _{\rho \rightarrow \infty}\left|\chi_{S_{p}^{o}}(q, \tilde{r})-\chi_{S_{o}^{o}}(q, \tilde{r})\right|,
$$

where $\chi_{A}$ means the indicator function of the set $A$. Then,

$$
S_{p}=\{(q, \tilde{r}) ; \min (0,\langle q, p\rangle) \leqq \tilde{r} \leqq(\max (0,\langle q, p)\rangle\} .
$$

Take $E_{R}=\left(S^{n-1} \times[-R, R], d \mu_{\infty}\right)$ and let $\mathscr{Y}$ be the Gaussian random measure associated with $E_{R}$. Set $\mathscr{X}_{R}$ be the limit $\rho \rightarrow \infty$ of $\overline{\mathscr{U}}_{R}^{-\rho}$;

$$
\mathscr{X}_{R} \equiv\left\{X(p) \equiv Y\left(S_{p}\right) ; p \in S^{n-1} \times[-R, R]\right\} .
$$

Then we obtain

Theorem 3. The projective limit $\mathscr{X}=\operatorname{proj}_{R \rightarrow \infty} \mathscr{X}_{R}$ is the Brownian motion parametrized in n-dimensional Euclidean space that corresponds to 
the usual metric. Moreover, the representation $X(p)=Y\left(S_{p}\right)$ is essentially equal to the one of N.N. Chentsov in 0-2.

Proof. Let us identify a hyperplane $H(\tilde{r}, q)=\{x ;\langle x, q\rangle=\tilde{r}\}$ with a point $(q, \tilde{r}) \in S^{n-1} \times \boldsymbol{R}_{+}$. Then the set

$$
\{H \in \mathscr{H} ; H \text { intersects the line segment } \overline{O p}\}
$$

is identified with

$$
\left\{(q, \tilde{r}) \in S^{n-1} \times R_{+} ; 0 \leqq \tilde{r} \leqq\langle q, p\rangle\right\} .
$$

Compare $\left(11^{\prime}\right)$ with (11) then we see that our new representation is just the Chentsov's representation of multiplicity 2.

1-4. Another examples of Brownian motions. The rest of this paper is devoted to the case of rotationally invariant metrics. But our formulation works for some of general metrics without rotationally invariance property.

ExAmple A. Let $\widetilde{\mathscr{H}}_{i}=\left\{h\right.$; hyperplanes in $\left.\boldsymbol{R}^{n}, h \perp \overline{O e_{i}}\right\}$, where $e_{i}$ is the unit vector parallel to the $i$-th coordinate, and let $\tilde{\mu}_{i}$ be the normalized uniform measure on $\widetilde{\mathscr{H}}_{i}$. Consider the measure space $(\tilde{\mathscr{H}}, \tilde{\mu})$, where $\widetilde{\mathscr{H}}=\widetilde{\mathscr{H}}_{1} \cup \widetilde{\mathscr{H}}_{2} \cup \cdots \cup \widetilde{\mathscr{H}}_{n}$ and $\tilde{\mu}=\tilde{\mu}_{1}+\tilde{\mu}_{2}+\cdots+\tilde{\mu}_{n}$. Define $S_{p}=$ $\{h \in \widetilde{\mathscr{H}}: h$ intersects $\overline{O p}\}$ and let $\mathscr{Y}$ be the random measure associated with $(\widetilde{\mathscr{H}}, \tilde{\mu})$. Then $\left\{X(p)=Y\left(S_{p}\right)\right\}$ is the Brownian motion that corresponds to the metric $d(x, y)=\sum\left|x_{i}-y_{i}\right|$.

EXAMPLE B. In the case of $\boldsymbol{R}^{2}$, set $\widetilde{\mathscr{H}}_{+}=\left\{h\right.$ : parallel with $\left.\overline{O\left(e_{1}+e_{2}\right)}\right\}$ and set $\widetilde{\mathscr{H}}_{-}=\left\{h\right.$; parallel with $\left.\overline{O\left(e_{1}-e_{2}\right)}\right\}$. The measure $\mu=(1 / \sqrt{2})\left(\mu_{+}+\right.$ $\left.\mu_{-}\right)$, the space $\widetilde{\mathscr{H}}=\widetilde{\mathscr{H}}_{+} \cup \widetilde{\mathscr{H}}_{-}$, the map $S_{p}$ and the random measure $\mathscr{Y}$ are all defined similarly to those in example A. Then the field $\left\{X(p)=Y\left(S_{p}\right)\right\}$ is the Brownian motion that corresponds to the metric

$$
d(x, y)=\max \left(\left|x_{1}-y_{1}\right|,\left|x_{2}-y_{2}\right|\right) .
$$

The author have been informed by private communications with professor A. Noda that for general metrics-for example $\ell^{p}$ metric on $\boldsymbol{R}^{n}$ and its $\alpha$-th power, $0<\alpha \leq 1$-the Chentsov type representation can be obtained.

\section{§2. Representation of Euclidean random field}

In this section we treat the Chentsov type representations of an important class of random fields-self similar Gaussian random fields-and 
a general class of Gaussian random fields with Euclidean invariance property.

2-1. Self-similar Euclidean random field.

Definition 3. A Gaussian system $\mathscr{V}^{\alpha}=\left\{V^{\alpha}(p) ; p \in \boldsymbol{R}^{n}\right\}$ is called a self-similar Euclidean random field $(S E R F)$ of index $\alpha$ if it satisfies

$$
\begin{gathered}
V^{\alpha}(O)=0, \\
V^{\alpha}(p)-V^{\alpha}(q) \stackrel{\mathscr{\Phi}}{=} N\left(0,|p-q|^{\alpha}\right) .
\end{gathered}
$$

The SERF exists if and only if $0<\alpha \leqq 2$ ([4]). It is easy to see that $|\cdot|^{\alpha}$ is a metric if and only if $0<\alpha \leq 1$. Therefore, the Chentsov type representation of SERF may be obtained in the case. In fact, we do form the representation in the case $0<\alpha<1$. (excluding the usual Brownian motion, $\alpha=1$ ).

Set $E=\boldsymbol{R}_{+} \times \boldsymbol{R}^{n}$ and introduce a coordinate in $E$ as

$$
x=\left(x_{0}, \underline{x}\right)=\left(x_{0}, x_{1}, x_{2}, \cdots, x_{n}\right), \quad x_{0}>0 .
$$

Consider a measure

$$
d \mu_{\alpha}(x)=M(\alpha) \cdot x_{0}^{\alpha-n-1} d x_{0} d \underline{x} \text { on } E,
$$

where $M(\alpha)$ is the normalizing constant which will be defined in (23). Let $\mathscr{Y}$ be the Gaussian random measure associated with $\left(E, \mu_{\alpha}\right)$. Define the following maps;

$$
\tilde{S}: R^{n} \longrightarrow \mathscr{B}(E) ; p \longrightarrow \tilde{S}_{p}=\left\{x ;|\underline{x}-p| \leqq x_{0}\right\}
$$

and

$$
S_{p}=S_{p} \triangle \tilde{S}_{o}
$$

Then,

TheOREM 4. For $0<\alpha<1$,

1) $\mu_{\alpha}\left(S_{p}\right)<\infty$,

2) $V^{\alpha}(p) \equiv Y\left(S_{p}\right)$ is the SERF of index $\alpha$.

Proof. 1)

$$
\mu_{\alpha}\left(S_{p}\right)=M(\alpha) \int_{0}^{\infty} r_{x_{0}}(t) x_{0}^{\alpha-n-1} d x_{0}=M(\alpha) \int_{0}^{\infty} x_{0}^{\alpha-1} r_{1}\left(\frac{t}{x_{0}}\right) d x_{0},
$$

where $t=|p|$. Because $r_{1}(t)=2 \sigma_{n}$ if $t \geqq 1$, 


$$
\left|\int_{0}^{1} x_{0}^{\alpha-1} r_{1}\left(\frac{t}{x_{0}}\right) d x_{0}\right|=\int_{1}^{\infty} y^{-\alpha-1} r_{1}(t y) d y \leqq 2 \sigma_{n} \int_{1}^{\infty} y^{-\alpha-1} d y<\infty, \quad \text { if } \alpha>0 .
$$

And because $r_{\rho}(t)=O\left(\rho^{n-1}\right), \rho \rightarrow \infty$, we get

$$
\left|\int_{1}^{\infty} r_{x_{0}}(t) x_{0}^{\alpha-n-1} d x_{0}\right| \leqq K \int_{1}^{\infty} x_{0}^{n-1} x_{0}^{\alpha-n-1} d x_{0}<\infty, \quad \text { if } \alpha<1 .
$$

Combining the two inequalities above, we get $\mu_{\alpha}\left(S_{p}\right)<\infty$ for $0<\alpha<1$.

2) $E\left|V^{\alpha}(p)-V^{\alpha}(q)\right|^{2}=E\left|Y\left(S_{p} \triangle S_{o}\right)-Y\left(S_{q} \triangle S_{o}\right)\right|^{2}=\mu_{\alpha}\left(S_{p} \triangle S_{q}\right)$. Note that the measure $\mu_{\alpha}$ is invariant under the action

$$
g x \equiv\left(x_{0}, g \underline{x}\right)
$$

of the group of solid motions $M(n)$. Then there is a positive function $s$ such that

$$
E\left|V^{\alpha}(p)-V^{\alpha}(q)\right|^{2}=s(p-q \mid) .
$$

For any $c>0$, we have

$$
\begin{aligned}
E\left|V^{\alpha}(c p)\right|^{2} & =M(\alpha) \int_{0}^{\infty} r_{x_{0}}(c t) x_{0}^{\alpha-n-1} d x_{0}=M(\alpha) \int_{0}^{\infty} x_{0}^{\alpha-1} r_{1}\left(\frac{c t}{x_{0}}\right) d x_{0} \\
& =c^{\alpha} M(\alpha) \int_{0}^{\infty} r_{1}\left(\frac{t}{x_{0}}\right) x_{0}^{\alpha-1} d x_{0}=c^{\alpha} E\left[V^{\alpha}(p)\right]^{2} .
\end{aligned}
$$

That is $s(c t)=c^{\alpha} s(t)=(c t)^{\alpha} s(1)$, and the fact $s(1)=1$ is derived from (23) and (24).

The SERF of index 1 is nothing but the ordinary Brownian motion and we have already obtained the representation of this type in 1-2. Consider the section $\tilde{S}_{p} \cap\left\{x_{0}=\rho\right\}$ of the set in (16). This is the $n$-ball of radius $\rho$. Thus we can see that the field $\mathscr{V}^{\alpha}$ of index $0<\alpha<1$ is represented by a superposition of the fields $\left\{\mathscr{U}^{\rho}\right\}$, that is, in an intuitive manner

$$
V^{\alpha}(p)=\int_{0}^{\infty} U^{\rho}(p) \rho^{(\alpha-n-1) / 2} d \rho .
$$

2-2. Euclidean random fields. Let us proceed to a more general class of random fields which can be obtained by superpositions.

Definition 4. A Gaussian system $\mathscr{X}^{r}=\left\{X^{r}(p) ; p \in \boldsymbol{R}^{n}\right\}$ is called a Euclidean random field (ERF) if it satisfies

$$
X^{r}(O)=0
$$

and 


$$
X^{r}(p)-X^{r}(q) \stackrel{\mathscr{s}}{=} N(0, r(|p-q|)),
$$

where $r$ is a positive continuous function.

The SERF in 2-1 is a special class of ERFs with $r(t)=t^{\alpha}$. In this subsection we consider the conditions under which the ERF has a Chentsov type representation.

Suppose that $X^{r}(p)$ is decomposed into a superposition of the independent family of ERFs $\left\{\mathscr{U}^{\rho}\right\}$, as $X^{r}(p)=\int U^{\rho}(p) \nu(\rho) d \rho$ with a positive measure $\nu(\rho) d \rho$. Let $d \mu(x)=\nu^{2}\left(x_{0}\right) d x_{0} d \underline{x}$ be a measure on $E=R_{+} \times R^{n}, S$. be the same in (16) and $\mathscr{Y}$ be the random measure which corresponds to $d \mu$. Then the assumption above is equivalent to the existence of the representation

$$
X^{r}(p)=Y\left(S_{p}\right) .
$$

The relation between $r$ and $\nu$ is given by

$$
r(t)=E\left[X^{r}(p)\right]^{2}=\int_{0}^{\infty} r_{\rho}(t) \nu^{2}(\rho) d \rho, \quad \text { where } t=|p|
$$

Thus if the function $r$ belongs to the image of the integral transform above, the ERF $\mathscr{X}^{r}$ has a Chentsov type representation.

The integral equation (21) can be solved as below;

THEOREM 5 (cf. [2]). If $\tilde{r}(t) \equiv(-d / d t)^{n+1} r^{\prime}(2 t) \geqq 0$ and $\int_{1}^{\infty} \tilde{r}(t) \cdot t^{n-1} d t<$ $\infty$, then the solution is obtained as

$$
\nu^{2}(\rho)=A \int_{\rho}^{\infty} \tilde{r}(t)\left(\frac{t^{2}}{\rho^{2}}=1\right)^{(n-1) / 2} d t
$$

$A$ being a positive constant.

We now consider another condition for the existence of the representation. We have already obtained a series of solutions of (21) in 2-1. Actually, set

$$
M(\alpha)=\int_{0}^{\infty} \rho^{\alpha-1} r_{1}\left(\frac{1}{\rho}\right) d \rho,
$$

then

$$
t^{\alpha}=\int_{0}^{\infty} \rho^{n} r_{1}\left(\frac{t}{\rho}\right) \frac{\rho^{\alpha-n-1}}{M(\alpha)} d \rho .
$$

Thus the density $\nu^{2}(\rho)$ in question which corresponds to the metric $r(t)=t^{\alpha}$ 
is expressible as $\rho^{\alpha-n-1} / M(\alpha)$. Let us consider again a superposition of these special solutions by a positive density $m(\alpha)$, say $X(p)=\int_{0}^{1} V^{\alpha}(p) m(\alpha) d \alpha$. That is, let us consider a measure $\mu$ on $E$ as

$$
d \mu(x)=\left(\int_{0}^{1} \frac{x_{0}^{\alpha-n-1}}{M(\alpha)} m^{2}(\alpha) d \alpha\right) d x_{0} d \underline{x} \text { and define }
$$

$$
X(p)=Y\left(S_{p}\right),
$$

where $\mathscr{Y}$ is again the random measure associated with $(E, \mu)$. Then, the corresponding function $r$ is

$$
r(t)=\int_{0}^{1} t^{\alpha} m^{2}(\alpha) d \alpha .
$$

Set $R(x)=r\left(e^{-x}\right)$. The Bernstein's theorem on Laplace transform tells us that the function $R(x)$ is completely monotone on $[0, \infty)$.

We then come to a class of functions $r$ satisfying the conditions below

A0) $\lim _{t \rightarrow 0} r(t)=r(0)=0$,

A1) $\tilde{d}(p, q)=r(|p-q|)$ defines a $C^{\infty}$-metric on $\boldsymbol{R}^{n}$.

A2) $R(x)=r\left(e^{-x}\right)$ is completely monotone.

By Bernstein's theorem there exists a positive measure $m^{2}(\alpha) d \alpha$ such that

$$
\begin{aligned}
R(x) & =\int_{0+}^{\infty} e^{-x \alpha} m^{2}(\alpha) d \alpha \quad \text { or equivalently } \\
r(t) & =\int_{0+}^{\infty} t^{\alpha} m^{2}(\alpha) d \alpha .
\end{aligned}
$$

Set $g(z)=\int_{0+}^{\infty} e^{i \alpha z} m^{2}(\alpha) d \alpha$ for $\operatorname{Im}(z) \geqq 0 . \quad$ Then,

Lemma 6. If $r(|p-q|)$ defines a metric on $\boldsymbol{R}^{n}$, then there are positive constants $c_{0}$ and $c_{1}$ such that $r(t) \leqq c_{0}+c_{1} t, t \in \boldsymbol{R}_{+}$.

Using this lemma, we prove that $\int_{0}^{\infty} t^{\alpha} m^{2}(\alpha) d \alpha \leqq c_{0}+c_{1} t$ for any $t$, that is $\left|\int_{0}^{\infty} e^{\alpha z} m^{2}(\alpha) d \alpha\right|<\infty$ for any $z \in C$. Thus the domain of definition of $g(z)$ can be extended to the whole complex plane.

Theonem 7. The function $g(z)$ is of inferior exponential type of order 1 , that is for any $\varepsilon>0$ there is a constant $C_{\varepsilon}$ such that

$$
|g(z)| \leqq C_{\varepsilon} e^{(1+\varepsilon)|\operatorname{Im}(z)|}, \quad \text { for any } z \in C .
$$


Proof.

$$
|g(z)| \leqq \int_{0+}^{\infty} e^{\alpha|\operatorname{Im}(z)|} m^{2}(\alpha) d \alpha=r\left(e^{|\operatorname{Im}(z)|}\right) \leqq c_{0}+c_{1} e^{|\operatorname{Im}(z)|} .
$$

Hence, we can choose the desirable constant $C_{\varepsilon}$ for any $\varepsilon>0$. Q.E.D.
Q

By virtue of the Paley-Wiener theorem, the support of the measure $m^{2}(\alpha) d \alpha$ is included in $(0,1]$. Finally we get;

THEOREM 8. If $r$ satisfies the conditions $\mathscr{A} 0$ )-Ad2) above, then the ERF $\mathscr{X}^{r}$ has Chentsov type representation,

$$
X^{r}(p)=Y\left(S_{p}\right)
$$

where the set $S_{p}$ is the same as in the case of SERF, and the corresponding measure $\mu_{r}$ is

$$
d \mu_{r}(X)=\left(\int_{0+}^{1} \frac{x_{0}^{\alpha-n-1}}{M(\alpha)} m^{2}(\alpha) d \alpha\right) d x_{0} d \underline{x} .
$$

N. B. The point mass at 1 corresponds to the original Chentsov representation.

2-3. Remark. In the remainder case of SERF of index $\alpha, 1<\alpha<2$, the covariance function $\sigma$ is positive definite but $|p-q|^{\alpha}$ is not a metric function. It is impossible to get Chentsov type representation. However we can get another type of representation-in the form of the Wiener integral.

Let $\mathscr{Y}$ be the Gaussian random measure associated with $\left(\boldsymbol{R}^{n}, d x\right)$ and $I(\cdot)$ be the Wiener integral with respect to $\mathscr{Y}$.

Theorem 9. Set $f(x) \equiv|x|^{(\alpha-n) / 2}$, for $\alpha \neq n$, and $\log |x|$ for $\alpha=n$, and set $F(t, x) \equiv f(x)-f(x-t), x, t \in \boldsymbol{R}^{n}$. Then for any $\alpha, 0<\alpha<2$,

1) $F(t, x) \in L^{2}\left(\boldsymbol{R}^{n}, d x\right)$, for any fixed $t$,

2) $X^{\alpha}(t) \equiv I(F(t, \cdot))$ is proportional to the SERF of index $\alpha$.

The proofs are quite similar to that of Theorem 4.

\section{§3. Invariance properties and representation}

The SERF $\mathscr{V}^{\alpha}$ has the following invariance properties:

Set

$$
Q_{g}(p) \equiv V^{\alpha}(g p)-V^{\alpha}(g O), \quad g \in M(n)
$$

and 


$$
Z^{u}(p) \equiv e^{-\alpha u / 2} V^{\alpha}\left(e^{u} p\right), \quad u \in \boldsymbol{R}^{1},
$$

then both $Q_{g}$ and $Z^{u}$ are again SERFs of the same index $\alpha$. It is obvious that the actions of $M(n)$ and the multiplications related to $Z^{u}$ form a group $G$.

Let us consider the relation between this invariance properties under $G$ and the representation of the Brownian motion. To simplify our discussion, we modify our representation of SERF as follows; set

$$
\tilde{V}^{\alpha}\left(S_{p}\right) \equiv \lim _{R \rightarrow \infty}\left\{Y\left(\tilde{S}_{p} \cap D_{R}\right)-Y\left(\tilde{S}_{o} \cap D_{R}\right)\right\}, \text { with } D_{R}=\left\{x=\left(x_{0}, \underline{x}\right) ; x_{0} \leqq R\right\},
$$

and write the left hand side as

$$
\tilde{Y}\left(F_{p}(x)\right) \text {, where } F_{p}(x)=\chi_{\tilde{s}_{p}}(x)-\chi_{\tilde{s}_{o}}(x) .
$$

N.B. In this section we consider invariance properties. If parallel transforms are considered, it is natural to treat stationary fields. But such a field $Y\left(S_{p}\right)$ does not exist. So we consider $\tilde{Y}\left(F_{p}(x)\right)$ as a substitute of $Y\left(S_{p}\right)$. Note that $F_{g p}(x) \neq F_{p}\left(g^{-1} x\right)$ for $g \in M(n), g \neq \mathrm{id}$.

The fields $\widetilde{V}^{\alpha}$ and $\mathscr{V}^{\alpha}$ has the same covariance. However, from the view point of group action, $\widetilde{\mathscr{V}}^{\alpha}$ behaves more naturally than $\mathscr{V}^{\alpha}$. In the rest of this paper, we use the modified field $\widetilde{\mathscr{V}}^{\alpha}$ instead of $\mathscr{V}^{\alpha}$. So in the definition (28) and (29), $V^{\alpha}$ has to be changed to $\tilde{V}^{\alpha}$.

Let us fix $\alpha$ between 0 and 1. It is obvious that the group $G$ is the subgroup of continuous point transforms of $\boldsymbol{R}_{+} \times \boldsymbol{R}^{n}$ which act as automorphisms on the family of subsets $\left\{\tilde{S}_{p} ; p \in \boldsymbol{R}^{n}\right\}$. Any element $\bar{g} \in G$ is written as $\bar{g}=(g, u)$, where $g$ is the part of solid motion and $u$ is the dilation part of $\bar{g}$. The action of $\bar{g}$ on the point $x \in \boldsymbol{R}_{+} \times \boldsymbol{R}^{n}$ is defined as

$$
\bar{g} x=\left(e^{u} x_{0}, e^{u} g \underline{x}\right) .
$$

Then we have;

THEOREM 10.

$$
Q_{g}(p)=\tilde{Y}\left(F_{g p}(x)\right)=\tilde{Y}\left(F_{p}\left(g^{-1} x\right)\right)-\tilde{Y}\left(F_{g o}(x)\right)
$$

and

$$
Z^{u}(p)=e^{-\alpha u / 2} \tilde{Y}\left(F_{p}\left(e^{-u} x\right)\right), \quad \text { a.a. } \omega .
$$

The theorem above describes the invariance properties of the SERF from the viewpoint of our representation and tells us why we have introduced $\tilde{Y}$. 
ADDEMDUm. SERF is called fractional Brownian motion and plays a central roll in the theory of fractals. The representation treated in 2-3 and its application were appeared in the paper of Mandelbrot [11].

\section{REFERENCES}

[1] Chentsov, N. N., Lévy's Brownian motion of several parameters and generalized white noise, Theory Probab. Appl., 2 (1957), 265-266.

[2] Deans, S. R., A unified Radon inversion formula, J. Math. Phys., 19 (1978), 23462349.

[ 3 ] Gangolli, R., Positive definite kernels on homogeneous spaces and certain stochastic processes related to Lévy's Brownian motion of several parameters, Ann. Inst. H. Poincaré Sect. B, 3 (1967), 121-226.

[ 4 ] Inoue, K. and Noda, A., Independence of the increments of Gaussian random fields, Nagoya Math. J., 85 (1982), 251-268.

[ 5 ] Lévy, P., Processus stochastiques et mouvement brownien, Gauthier-Villars (1965).

[6] Lifshits, M. A., On representation of Lévy's field by indicators, Theory Probab. Appl., 24 (1979), 629-633.

[ 7 ] Molcan, G. M., Markov property of Lévy's fields on spaces of constant curvature, Dokl. Akad. Nauk CCCP, tom. 221 (1975), 1276-1279.

[8] Noda, A., Integral transformation, associated with Lévy's Brownian motion, Tech. Rep. Univ. Erlangen (1984).

[9] Pflug, G., A statistically important Gaussian process, Stochastic Process. App]., 13 (1982), 45-57.

[10] Takenaka, S., Kubo, I. and Urakawa, H., Brownian motion parametrized with metric space of constant curvature, Nagoya Math. J., 82 (1981), 131-140.

[11] Mandelbrot, B., Fonctions aléatoires plurei-temporelles; approximation poissonienne de cas brownien et généralisations, C.R. Acad. Sc. Paris, 280A (1975), 1075-1078.

[12] Noda, A., Generalized Radon transform and Lévy's Brownian motion I, II, Nagoya Math. J., 105 (1987), 71-87, 89-107.

[13] Ossiander, M. and Pyke, R., Lévy's Brownian motion as a set-indexed process and a related central limit theorem, Stochastic Process. Appl., 21 (1985), 133-145.

Department of Mathematics

Nagoya University

Chikusa-ku, Nagoya 464

Japan 